\title{
The role of the mirror-image reversal effect in reducing the peak shift and area shift along the angularity dimension
}

\author{
WILLIAM D. KLIPEC, LARRY LINDBLOM, and LINDA LINDBLOM \\ Drake University, Des Moines, Iowa 50311
}

\begin{abstract}
Pigeons trained monocularly and tested with the trained eye along the angularity dimension were found to show enhanced area shifts when compared to pigeons trained and tested binocularly. Pigeons trained monocularly and tested with the untrained eye showed reversed peak shifts in the direction of $\mathbf{S}-$. These findings are consistent with the hypothesis that the mirror image of $\mathrm{S}-$ in angularity gradients functions to reduce the likelihood of the peak shift and area shift along the angularity dimension in pigeons, and question the use of the angularity dimension for intradimensional discrimination training and generalization testing.
\end{abstract}

The peak shift is a phenomenon, first reported by Hanson (1959), which may be described as a shift in the modal response category during generalization testing from the stimulus value correlated with reinforcement during training $(S+)$ to a value beyond $\mathrm{S}+$ in a direction further removed from the stimulus correlated with extinction or a relatively less dense schedule of reinforcement $(\mathrm{S}-)$. The enhancement in responding occurs not only to the peak-shift stimulus, but also to all stimuli on the peak-shift side of the gradient. Terrace (1964) called this enhancement the area shift and suggested that it represented the same process underlying the peak shift. The peak shift has been found following a variety of discrimination training procedures which involve a reduction in responding and/or reinforcement density to one stimulus in a multiple schedule (see Purtle, 1973, for a review of this leterature).

The peak shift is quite pervasive, having been demonstrated along the dimensions of line tilt with pigeons (Bloomfield, 1969) and monkeys (Ray, 1967); wavelength with pigeons (Hanson, 1959), goldfish (Yarczower \& Bitterman, 1965), and humans (Doll \& Thomas, 1967); floor position with pigeons (Riccio, Urda, \& Thomas, 1966) and rats (Lyons, Klipec, \& Eirick, 1973); and with auditory stimuli, using rats (Pierrel \& Sherman, 1960). Nonetheless, the majority of peak-shift studies in the literature have used pigeons and the dimensions of either angularity or wavelength.

In examining the data from a number of studies in our laboratory, we noticed that the probability of a peak shift and the magnitude of the area shift were

Some of the data from this paper were presented at the Rocky Mountain Psychological Convention, Phoenix, 1976. Requests for reprints should be sent to the first author. Larry Lindblom is now at McMaster University, Hamilton, Ontario, Canada. considerably smaller when using the angularity rather than wavelength dimension. On discriminations of equivalent difficulty for the pigeons, about $25 \%$ exhibited the peak shift along the angularity dimension, while about $85 \%$ showed the peak shift along the wavelength dimension. Similar difficulties in obtaining line-angle peak shifts have been reported, both informally by other laboratories, and in the literature (Hearst, 1968). The purpose of the present experiment was to investigate the reasons for the decreased likelihood of the peak and area shift along the angularity dimension.

Earlier research by Mello (1965a, 1965b) has shown that pigeons trained monocularly to respond to an oblique line will show a gradient with modal responding at the training stimulus when tested with the trained eye, but show a gradient with a maximum value at the mirror image of the training stimulus when tested with the untrained eye. Thomas, Klipec, and Lyons (1966) found that the interaction between the training stimulus and its mirror image in pigeons was not restricted to monocular training and testing. Following binocular training with an oblique line as $\mathrm{S}+$ and binocular generalization testing, Thomas et al. (1966) found bimodal generalization gradients with a peak at the training stimulus and a secondary peak at its mirror image.

Subsequent research in this area has attempted to delineate stimulus parameters of the mirror-image reversal effect by investigating the phenomenon with vertically split keys (Beale \& Corballis, 1968) and radial stimuli (Watanabe, 1975; Watanabe \& Ogawa, 1973). Watanabe (1975) found a small proportion of subjects showing peak shifts, but a clear mirrorimage reversal of angularity gradients following intradimensional monocular training with radial stimuli. However, the $S+$ in the Watanabe (1975) experiment was always an oblique line, while $S-$ was 
either a vertical line or one of several oblique lines, making comparisons to the typical peak-shift experiment difficult. In the typical peak-shift experiment involving angular stimuli, the discrimination has been between an oblique $\mathrm{S}-$ and a vertical $\mathrm{S}+$, with both stimuli forming the diameter, rather than the radius of the pecking key. Using diametric stimuli with a vertical $S+$ and an oblique $S-$, one of the stimuli to which the peak shift would be expected to occur is the mirror image of $\mathrm{S}-$. Hanson (1961) has shown that the peak shift along the wavelength dimension can be eliminated by using intradimensional discrimination training with an $\mathrm{S}-$ on each side of $\mathrm{S}+$. This training results in a steepening of the gradient around $\mathrm{S}+$ without the development of the peak and area shift typically found using a single $\mathbf{S}-$. When using the angularity dimension with a single oblique $\mathrm{S}-$ and a vertical $\mathrm{S}+$, stimuli on the peak-shift side of the gradient are all mirror images of $S-$ and the stimuli surrounding $S-$. If mirror-image reversal effects occur, these conditions are functionally similar to Hanson's (1961) procedure with two S - stimuli. Under these conditions, the tendency for the peak-shift stimulus to control a high rate of responding could be reduced by the tendency for it as the mirror image of $\mathrm{S}$ - to control a low rate of responding. This opposition of control of high and low rates of responding would not occur on dimensions where mirror-image reversals do not occur, such as wavelength, and could account for the reduced probability of obtaining peak shifts along the dimension of angularity relative to wavelength in pigeons.

The hypothesis that the rate of responding to the peak-shift stimulus is attenuated by its also being the mirror image of $\mathrm{S}$ - can be easily tested with monocular discrimination training and testing. If the hypothesis is correct, pigeons trained monocularly and tested with the trained eye should be more likely to show the peak shift and show stronger area shifts than pigeons trained and tested binocularly, since the mirror-image effect would be eliminated by monocular training and testing. Moreover, pigeons trained monocularly and tested with the untrained eye should show a peak shift toward the $\mathrm{S}$ - side of the gradient, i.e., in the direction opposite to that obtained with the trained eye. The following experiment was conducted to test these hypotheses.

\section{METHOD}

\section{Subjects}

Fifteen experimentally naive roller pigeons (Columba liva) from a local breeder were used in the experiment. Upon arrival at the laboratory, each subject was individually housed with free access to food, grit, and water. The animals were reduced to $75 \%-80 \%$ of their free-feeding weights by restricted feedings of $3 \mathrm{~g}$ daily. Water and grit were freely available throughout the experiment.

\section{Apparatus}

Training and testing were conducted in three standard operant conditioning chambers, constructed in the laboratory but similar in every respect to commercially available units. The intelligence panels were housed in 58-qt Coleman ice chests, which were modified to accept cables and equipped with exhaust fans. The Ferstertype pecking keys were transilluminated with I.E.E. display cells capable of displaying white $.32-\mathrm{cm}$-wide lines forming diameters of a $2.54-\mathrm{cm}$-black surround. The stimuli used were $140^{\circ}$ (a $50^{\circ}$ counterclockwise rotation from vertical), $130^{\circ}, 120^{\circ}, 110^{\circ}, 100^{\circ}$, $90^{\circ}$ (vertical), $80^{\circ}, 70^{\circ}, 60^{\circ}, 50^{\circ}$, and $40^{\circ}$. All recording and programming was accomplished with electromechanical devices. A Gerbrands white-noise generator provided a continuous masking noise to the chambers.

\section{Procedure}

Several procedures for rendering the pigeon monocular were investigated in pilot studies. Hoods and eye patches were observed to shift considerably during pecking. Ophthalmic drops used to occlude vision in one eye by interfering with cilliary accommodation were unsatisfactory, since pigeons showed some evidence of tolerance after prolonged application. In this experiment, pigeons were rendered monocular by suturing the eyelid shut with a continuous external suture. To eliminate the necessity for repeated resuturing of the delicate eyelid tissue and to avoid the need for repeated generalization testing, a between-groups design was selected.

After attaining $75 \%$ of its free-feeding weight, each pigeon entered Session 1 of the experiment. Sessions 1 through 25 represented preliminary training, and procedures were identical for all pigeons. The purpose of the preliminary training was to provide a gradual transition from continuous reinforcement to a variableinterval 1-min (VI 1-min) schedule of reinforcement and establish control by the vertical line $(S+)$ prior to suturing the eyelids and introducing an oblique line $(\mathbf{S}-)$. This was done to minimize the length of time the sutures needed to remain intact.

In Session 1, the key was illuminated with a white light and both the keylight and houselight were on continuously. Pigeons were magazine and keypeck trained by successive approximations and allowed to earn 50 continuous reinforcements $(3 \mathrm{sec}$ access to mixed grain) following the first keypeck. In Session 2, and all subsequent sessions, including generalization tests, a stimulus presentation consisted of a 50-sec on-time during which the key-and houselights were on, aiternated with a 10-sec blackout during which the key- and houselights were off and the key was inoperative. In Sessions 2 aqnd 3, pigeons earned 50 continuous reinforcements for responding. Sessions 4 and 5 were run for $15 \mathrm{~min}$ with VI 15-sec and VI 30-sec reinforcement schedules in effect, respectively. Sessions 6 through 15 were run for $30 \mathrm{~min}$ with responding maintained on a VI 1 -min schedule. Sessions 16 through 25 were identical to Sessions 6-15, except that the white keylight was replaced with the white vertical line on a black surround $(S+)$. On the day following Session 25, the pigeons were randomly divided into three groups of five each. Two of the groups were subsequently trained monocularly and tested with the trained (TE group) and untrained eyes (UE group), respectively. The third group (BIN) was subsequently trained and tested binocularly. Pigeons in the TE and UE groups were first given $.25 \mathrm{mg}$ atropine and then anesthetized with $35 \mathrm{mg} / \mathrm{kg}$ of intramuscular pentobarbital sodium. Intramuscular administrations of $60 \mathrm{mg}$ of chloral hydrate were given as needed to maintain anesthetic level. A continuous external suture spaced at $1 \mathrm{~mm}$ closed the external eyelid of the left eye. As a control for residual drug effects, the pigeons in the BIN group were anesthetized with no surgical intervention.

Following a 24-h recovery period, discrimination training was introduced. Training was identical for pigeons in all groups. Sessions had previously been separated by $24 \mathrm{~h}$, but were now conducted at 12 -h intervals to minimize the amount of time that the sutures needed to remain in place. Sessions were $30 \mathrm{~min}$ in 
duration, with the vertical line $\left(\mathbf{S}_{+}\right)$on the key for 15 presentations, randomly alternated with 15 presentations of a line tilted $30^{\circ}$ counterclockwise $(S-)$. Responding during $S+$ presentations was maintained on a VI 1-min schedule, while extinction was in effect during $S$ - presentations. All subjects were run to a criterion of two consecutive sessions of a $10: 1$ ratio of $S+: S-$ responding or a maximum of 10 sessions. At this time, UE pigeons were anesthetized, the sutures were removed from the left eye, and the right eyelid was sutured shut. As a control for residual drug effects, all pigeons in the TE and BIN groups were anesthetized and allowed to recover. Following a 24 -h recovery period, all pigeons were tested for generalization to 11 line-angle stimuli which varied in $10^{\circ}$ steps from $140^{\circ}$ to $40^{\circ}$. The generalization test was conducted in extinction, with all stimuli randomized within a series, and four different series presented to each bird. Prior to the test, each bird received a warm-up period during which $\mathrm{S}+$ was presented 10 times with a VI 1 -min schedule of reinforcement in effect.

\section{RESULTS}

The total responses emitted to each stimulus during generalization testing was computed for each pigeon. The absolute generalization gradients for each pigeon are presented for Figure 1 for each of the three groups. Inspection of the gradients for pigeons in the TE group reveals that three of the five subjects showed a peak shift in the direction away from $\mathrm{S}-$, with the remaining subjects (TE 1 and 5) showing area shifts. In the BIN group, two of the five subjects show a peak shift in the direction away from $\mathrm{S}-$; the remaining pigeons (BIN 1, 3, and 5) showed no peak shifts and somewhat reduced area shifts compared to the TE pigeons. Four of the five subjects in the UE group showed peak shifts in the opposite direction, i.e., toward the $\mathrm{S}$ - side of the gradient. While the remaining pigeon (UE 4) showed modal responding at the $40^{\circ}$ stimulus, there was a substantial enhancement of responding to the $S$ - side of the gradient when compared to subjects in other groups.

One particularly interesting finding evident in Figure 1 is the reduced responding to the $70^{\circ}$ stimulus, which is $10^{\circ}$ removed from the mirror image of $\mathrm{S}-$, that occurred for several pigeons. The three birds in the BIN group that showed this reduction BIN 1, 3, and 5) also failed to show the peak shift. While this finding is consistent with the hypothesis that the mirror image of $S-$ attenuates responding to the peak-shift stimuli, similar reductions in responding to the $70^{\circ}$ stimulus in the TE group were not systematically associated with the absence of the peak shift. Note that for the three birds showing reduction to the $70^{\circ}$ stimulus in the TE group, two showed the peak shift (TE 2 and 4) while one did not (TE 5).

The pigeons in the UE group that showed the reversed peak shift (UE 1, 2, 3, and 5) showed reduced responding in the area of $\mathrm{S}-$ and its mirror image but the location of the reduced responding varied across pigeons. Note that, like the three TE and three
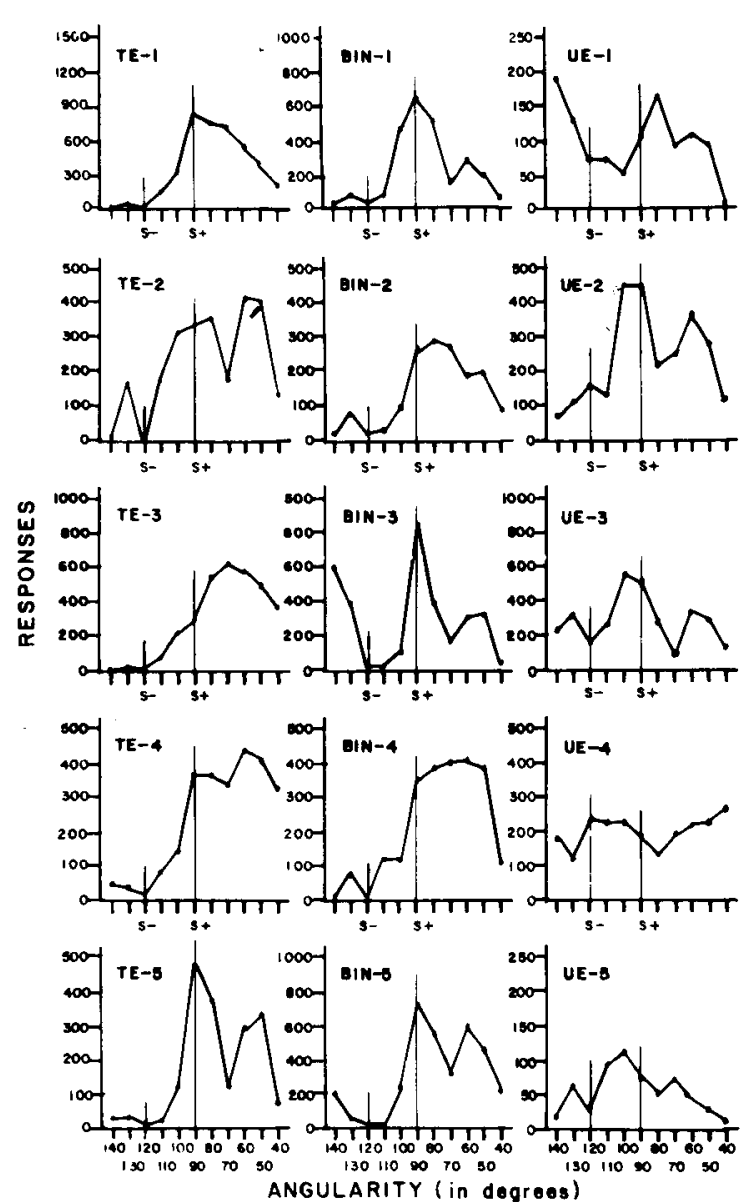

Figure 1. Absolute generalization gradient for pigeons trained and tested binocularly (BIN), or trained monocularly and tested with the trained eye (TE) or untrained eye (UE).

BIN subjects mentioned earlier, none of these four pigeons produced a minimum at the mirror image of $\mathrm{S}-\left(60^{\circ}\right)$. All UE birds displayed reduced responding at either the $70^{\circ}$ or the $80^{\circ}$ stimulus. Thus, it would appear that the mirror-image transfer effect was not veridical, since none of the pigeons that showed this minimum value did so at the $60^{\circ}$ stimulus. Moreover, while a dip at or near the mirror image of $\mathrm{S}$ - in the binocular group was expected, the occurrence of the dip at both the $\mathrm{S}$ - and near its mirror image in three of the TE birds and all of the UE birds suggests that the mirror-image transfer effects may not have been completely eliminated by the monocular training.

Mean relative gradients were constructed for each of the three groups by computing the percent of total responses to each stimulus for each pigeon and then computing the mean percent of total responses to each stimuli. Figure 2 presents these gradients. Inspection of Figure 2 reveals a peak shift in the direction away from $\mathrm{S}-$ for the TE group, no peak shift for the BIN group, and a peak shift toward the $\mathrm{S}$ - side of the gradient for the UE group. Moreover, 


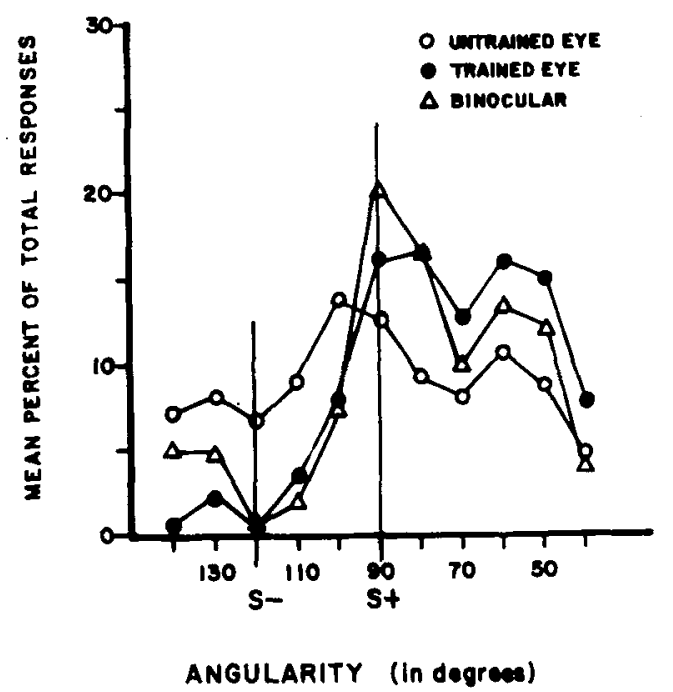

Figure 2. Mean relative generalization gradients for pigeons trained and tested binocularly (BIN) or trained monocularly and tested with the trained eye (TE) or untrained eye (UE). There were five pigeons in each group.

there is the predicted systematic reduction in the area shift for the UE, BIN, and TE groups, respectively, with the UE group showing a substantially higher percentage of responses on the $S$ - side of the gradient.

In order to assess the reliability of these differences, a 2 by 3 mixed analysis of variance with simple main effects tests was conducted on the percent of total responses on the $S-$ side of the gradient (the $140^{\circ}$, $130^{\circ}, 120^{\circ}, 110^{\circ}$, and $100^{\circ}$ stimuli) and the area shift (the $80^{\circ}, 70^{\circ}, 60^{\circ}, 50^{\circ}$, and $40^{\circ}$ stimuli) across the three groups. These analyses revealed that there was a reliable difference in percent of total responses among the three groups on both the $\mathrm{S}-$ side of the gradient $[F(2,12)=11.56, p<.01]$ and area shift $[\mathrm{F}(2,12)=10.33, \mathrm{p}<.01]$. The predicted increase in the area shift for the trained-eye group compared to the binocular group was significant $[\mathrm{t}(12)=2.06$, $\mathrm{p}<.05]$, using an a priori $\mathrm{t}$ test. The remaining comparisons were planned, but not orthogonal, and were tested with Dunn's procedure. The area shift for the trained-eye group and binocular group was significantly greater than the untrained-eye group $[\mathrm{t}(12)=4.47$, $\mathrm{p}<.01$, and $\mathrm{t}(12)=2.82, \mathrm{p}<.01$, respectively]. On the $\mathrm{S}$ - side of the gradient, the untrained-eye group showed significantly greater responding than either the trained-eye or binocular groups $[\mathrm{t}(12)=5.42, \mathrm{p}<.01$, and $\mathfrak{t}(12)=4.35, \mathrm{p}<.01$, respectively], but the trained-eye and binocular groups were not significantly different. Using these three procedures, the probability of a Type I error in the entire collection of comparisons is .05 or less.

\section{DISCUSSION}

The enhancement of the peak shift and area shift found in the UE subjects compared to the BIN subjects is consistent with the hypothesis that the angularity peak shift is attenuated by the mirrorimage reversal effect. Moreover, the finding of a reversed peak and area shift in the UE subjects replicates the Watanabe (1975) finding that the mirror-image reversal effect is active following intradimensional discrimination training, and extends it to include conditions typically found in binocular peak-shift experiments. The reduction of responding to the $70^{\circ}$ stimulus in all three BIN pigeons that failed to show the peak shift is also consistent with the hypothesis that the mirror image of $\mathrm{S}$ - exerts a local inhibitory effect in the region of the peakand area-shift stimuli. However, this evidence is weakened by the failure of this local effect to eliminate the peak shift in two of the three TE subjects in which the effect occurred. Moreover, it is difficult to understand the reduction of responding to the $70^{\circ}$ stimulus that occurred in the TE group, since mirrorimage reveral effects should have been eliminated by the monocular training conditions.

The data from the UE group also suggest that the monocular training may have produced only a partial monocular effect. All five of the UE birds, like the three TE birds mentioned above, showed a response decrement at both $\mathrm{S}$ - and near its mirror image. A complete mirror-image reversal effect would predict the elimination of inhibitory control at $\mathrm{S}$ - and the complete transfer of inhibitory control to its mirror image. Moreover, the UE gradient is not the complete mirror image of the TE gradient, which would be predicted from a complete mirror-image reversal effect. Rather, the gradient shows a substantially smaller area shift to the $\mathrm{S}$ - side of the gradient and a substantially greater amount of responding around the mirror image of $\mathrm{S}-$ than would be expected.

It is possible that the reduced responding to the $70^{\circ}$ and $80^{\circ}$ stimuli was due to some idiosyncratic effect of the stimulus projectors, but this is unlikely, since the effect was distributed across all three chambers, and the projectors were routinely checked before and after training and test sessions. It is also possible that the persistent dip on both sides of the gradient was due to a tendency for pigeons to show reduced responding to those stimuli. While this could account for the reduced responding to the $70^{\circ}$ and $80^{\circ}$ stimuli in the TE and BIN birds, it could not also account for the UE birds showing greater responding to those stimuli than would have been expected. Finally, it is possible that partial monocular effects could have been produced if a gap developed in the sutured eyelid that emitted some light and formed a partial image of the stimulus. This explanation is rejected, however, since the eyelids were closely sutured and were inspected for gaps before all runs and tests. Without exception, the suture line was found to be not only intact, but encrusted with a dried mucus secretion which would tend to seal any gaps that might have been too small to detect. 
Finding partial monocular effects following monocular training and testing is not without precedence. Watanabe (1975) reported finding bimodal excitatory gradients following monocular single stimulus training to an oblique radial stimulus in both TE and UE subjects. Watanabe's use of goggles held in place by colloidin to produce monocular subjects makes the formation of a partial image an unlikely explanation for the effects. While the data on the occurrence of partial monocular effects is not conclusive, it is highly suggestive, and may have implications for understanding the mechanism for the mirror-image transfer phenomena.

The traditional view of the mechanism for the mirror-image reversal effect has been based on the neuroanatomical structure of the pigeon's visual system. Since the pigeon has $100 \%$ decussation of the optic nerves at the chiasma, all of the visual information from one eye is projected to the contralateral hemisphere. This view holds that the reversal of the image occurs in the interhemispheric transfer of information between the optic tecta. Given the assumption that the sutured eyelids prevented stimulation of the retina by light energy passing through the sutured eye, the evidence for a partial monocular effect in this experiment is puzzling.

An alternative interpretation is that the mirrorimage reversal effect may simply be due to the anatomical arrangement of the pigeon's eyes rather than a neuroanatomical mechanism. ${ }^{1}$ That is, since the pigeon has lateral eyes, the retinas of the two eyes are opposed in parallel fashion and separated by only a small amount (about 1-2 $\mathrm{mm}$ ) of tissue. Thus, light energy focusing on one retina could stimulate the contralateral retina, but the image would be reversed from normal since the light energy would be striking the retina from the opposite direction. This is possible since the pigeon is slightly hypermetropic, i.e., tends to have a focal point behind the retina (see Pearson, 1972; Sturkie, 1965). This alternative interpretation is appealing, since suturing the eyelids would have no effect on stimulation of the retina by an image focused through the contralateral eye. Clearly, further research is needed to investigate both the occurrence of partial monocular effects and the mechanism for the mirror-image reversal effects before the phenomenon can be fully understood.

While the evidence for partial monocular effects is not conclusive, if such effects did occur, the present data underestimate the extent to which the mirror image of S - interacts with stimuli on the peak-shift side of the gradient. The present data strongly suggest that mirror-image reversal effects may act to reduce the likelihood of obtaining peak shift and area shift along the angularity dimension in the context of binocular training and testing. Thus, research on the peak shift and related phenomena should be conducted using dimensions other than angularity. Moreover, studies in the literature as well as lines of research which have been discontinued due to a failure to obtain peak shift or related phenomena along the angularity dimension with pigeons should be reexamined in the context of these findings.

\section{REFERENCES}

Beale, I. L., \& Corballis, M. C. Peak shift: An explanation for interocular mirror image reversal in pigeon. Nature, 1968, 220, 82-83.

Bloomfield, T. M. Behavioral contrast and the peak shift. In R. M. Gilbert \& N. E. Sutherland (Eds.), Animal discrimination learning. London: Academic Press, 1969.

Doll, T. J., \& Thomas, D. R. Effects of discrimination training on stimulus generalization for human subjects. Journal of Experimental Psychology, 1967, 75, 508-512.

Hanson, H. M. The effects of discrimination training on stimulus generalization. Journal of Experimental Psychology, 1959. 58, 321-334.

Hanson, H. M. Stimulus generalization following three stimulus discrimination training. Journal of Comparative and Physiological Psychology, 1961, 54, 181-185.

Hearst, E. Discrimination learning as the summation of excitation and inhibition. Science, 1968, 162, 1303-1306.

Lyons, J., Klipec, W. D., \& Eirick, R. The effects of chlorpromazine on the peak shift in the male albino rat. Physiological Psychology, 1973, 1, 165-168.

Mello, N. K. Inter-hemispheric reversal of mirror-image oblique lines following monocular training in pigeons. Science, 1965 , 148, 252-254. (a)

Melzo, N. K. Reply to Cumming, Siegel, and Johnson. Science, $1965,194,1519-1520$. (b)

Pearson, R. The avian brain. New York: Academic Press, 1972.

Pierrel, R., \& Sherman, J. G. Generalization of auditory intensity following discrimination training. Journal of the Experimental Analysis of Behavior, 1960, 3, 312-322.

Purtle, R. B. Peak shift: A review. Psychological Bulletin, 1973, 80, 408-421.

RAY, B. A. The course of acquisition of a line tilt discrimination by thesus monkeys. Journal of the Experimental Analysis of Behavior, 1967, 10, 17-33.

Riccio, D. C., Urda, M., \& Thomas, D. R. Stimulus control in pigeons based on proprioceptive stimuli from floor inclination. Science, 1966, 153, 434-436.

Sturkie, P. D. Avian physiology. Ithaca, New York: Cornell University Press, 1965.

Terrace, H. S. Wavelength generalization after discrimination learning with and without errors. Science, 1964, 144, 78-80.

Thomas, D. R., Klipec, W., \& Lyons, J. Investigation of mirror image transfer effect in pigeons. Journal of the Experimental Analysis of Behavior, 1966, 9, 567-570.

WATANABE, S. Interoculat transfer of generalization along linetilt dimension in pigeons: A separation of three types of symmetric stimuli. Japanese Psychological Research, 1975. $17,3,133-140$.

Watanabe, S., \& Ogawa, T. An experimental analysis of mirror image reversal effects in pigeons. The Annual of Animal Psychology, 1973, 23, 1-13.

YARCZOWER, M., \& BitTerman, M. E. Stimulus generalization in the goldfish. In D. Mostofsky (Ed.), Stimulus Generalization. Stanford, California: Stanford University Press, 1965.

\section{NOTE}

1. The idea that the mirror-image reversal effect was due to the lateral positioning of the eyes was suggested by Dudley Klopfer in 1975 in a personal communication.

(Received for publication August 24, 1977; revision accepted August 2, 1978.) 